\title{
Formulas for Bivariate Hyperosculatory Interpolation
}

\author{
By Herbert E. Salzer
}

\begin{abstract}
For a given function $f(x, y)$, bivariate hyperosculatory interpolation formulas are obtained by employing a suitably constructed binary nic $p_{n}(x, y)$ that is fitted to the values of $f(x, y)$ and its first and second partial derivatives at the $m$ points $\left(x_{i}, y_{i}\right)$ of a rectangular $h \times k$ Cartesian grid, where $\left(x_{i}, y_{i}\right)=\left(x_{0}+p_{i} h, y_{0}+q_{i} k\right), p_{i}$ and $q_{i}$ are small integers $\geqq 0, i=0(1) m-1, m \geqq 2$. In terms of the variables $(p, q)$, where $x=$ $x_{0}+p h, y=y_{0}+q k$ (and $f(x, y)=F(p, q)$ ), we have $p_{n}(x, y)=P_{n}(p, q)$. Often, for $P_{n}(p, q)$ having a specified desirable form, this problem turns out to be insoluble for every configuration of the points $\left(x_{i}, y_{i}\right)$. When this is not the case, it generally requires considerable investigation to find a practical configuration of points $\left(x_{i}, y_{i}\right)$ for which there is a solution of the form $P_{n}(p, q)$. Formulas are found for choices of $P_{n}(p, q)$, and soluble configurations of points $\left(x_{i}, y_{i}\right)$, that have dominant remainder terms in
\end{abstract}

$$
h^{r} k^{\circ} f_{x \ldots x}(r \text { times }) y \ldots y(e \text { times })\left(x_{0}, y_{0}\right)
$$

whose orders $r+s$ are as high as possible. Three two-point formulas, two three-point formulas and one four-point formula, including all remainder terms through the order

$$
r+s=\left[\begin{array}{ll}
n, & \text { for } m=2 \\
n+1, & \text { for } m=3,4
\end{array}\right),
$$

are given here in convenient matrix form.

1. Introduction. This present article is concerned with some formulas for $m$-point bivariate hyperosculatory interpolation over an $h \times k$ rectangular Cartesian grid, where we interpolate for $f(x, y)$ by means of a binary nic $p_{n}(x, y)$, which together with its first and second partial derivatives at $\left(x_{i}, y_{i}\right)$ agrees with $f_{i} \equiv f\left(x_{i}, y_{i}\right)$, $f_{x_{i}} \equiv f_{x}\left(x_{i}, y_{i}\right), f_{y i} \equiv f_{y}\left(x_{i}, y_{i}\right), f_{x x_{i}} \equiv f_{x x}\left(x_{i}, y_{i}\right), f_{x y_{i}} \equiv f_{x y}\left(x_{i}, y_{i}\right)$ and $f_{y y_{i}} \equiv f_{y y}\left(x_{i}, y_{i}\right)$, $x_{i}=x_{0}+p_{i} h, y_{i}=y_{0}+q_{i} k$, where $p_{i}$ and $q_{i}$ are small integers $\geqq 0, i=0(1) m-1$, and $x=x_{0}+p h, y=y_{0}+q k$. Such formulas might be specially convenient when $f(x, y)$ is the solution of a second-order partial differential equation where some, or even all, of the first and second partial derivatives are readily available at $\left(x_{i}, y_{i}\right)$, either as a byproduct of a numerical solution of the equation, or from the equation itself.

2. Previous Related Work. To review briefly the situation for osculatory and hyperosculatory interpolation up to the present investigation, we recall first the very widely known fact that for a single variable we can always find a unique polynomial of degree $n$, say $P_{n}(x)$, such that at any $m$ points $x_{i}, i=O(1) m-1$, regularly or irregularly spaced, real or complex, we have $P_{n}^{(i i)}\left(x_{i}\right)=f^{(i)}\left(x_{i}\right), j_{i}=0(1) k_{i}$, $\sum_{i=0}^{m-1} k_{i}+m=n+1$. For two variables, even for ordinary interpolation, say in

Received December 22, 1969, revised July 2, 1970.

AMS 1970 subject classifications. Primary 65D05, 41A05; Secondary 41A10, 41A63.

Key words and phrases. Bivariate hyperosculatory interpolation, bivariate interpolation, hyperosculatory interpolation, Cartesian interpolation, interpolation, remainder formulas. 
fitting a binary nic like $a+b x+c y+d x^{2}+e x y+f y^{2}+\cdots+r x^{n}+s x^{n-1} y+$ $\cdots+t y^{n}$ to $f\left(x_{i}, y_{i}\right), i=0,1, \cdots,(n+1)(n+2) / 2-1$, there is the restriction that the determinant $\left|1, x_{i}, y_{i}, x_{i}^{2}, x_{i} y_{i}, y_{i}^{2}, \cdots, x_{i}^{n}, x_{i}^{n-1} y_{i}, \cdots, y_{i}^{n}\right| \neq 0$, which appears to be just mildly restrictive. It was the detailed investigation of bivariate osculatory interpolation, i.e., fitting a binary polynomial to $f_{i}, f_{x_{i}}$ and $f_{y_{i}}$, $i=0(1) m-1$, that brought out some surprising results about the insolubility of the problem under a wide variety of conditions [1]. Thus there were some cases where a binary polynomial of prescribed form fails for any choice of points $\left(x_{i}, y_{i}\right)$, and in other cases where it may fail for just certain special configurations of the points $\left(x_{i}, y_{i}\right) *$ To obtain solutions in the former cases, it was necessary to slightly distort a natural looking choice of a binary polynomial, by leaving out a lower degree term and adding one of higher degree. For binary polynomials that were not generally insoluble, it was often found that the closest and most symmetrical configurations of $\left(x_{i}, y_{i}\right)$, e.g., : . for three points, or : : for four points, did not have a solution, and a considerable amount of searching was necessary in order to find the closest configurations that were soluble. But for the present problem of bivariate hyperosculatory interpolation, it turns out that these difficulties of finding a suitable binary polynomial, and also configurations of $\left(x_{i}, y_{i}\right)$ that have a solution, are so magnified that there is much less leeway in the selection of workable formulas.

The foregoing discussion indicates how we may be misled in expecting certain properties in univariate interpolation to hold in multivariate interpolation. Thus all the standard forms of the interpolation polynomial in one variable, e.g., Lagrangian, Gregory-Newton, Newton-Bessel, Everett, and even Newton's general divided difference formula, have mixed confluent forms of every variety. But for bivariate interpolation, there is no unambiguous definition of a formula for preassigned confluent points of specified multiplicity, since in the process of the confluence of points in the two-dimensional plane, the direction of approach determines the form of the result. Furthermore, even when the direction of approach in the confluence of points is specified, in general certain limiting confluent forms may not exist.**

In connection with bivariate interpolation, the terminology "irregularly-spaced points in two dimensions" in most books on classical numerical analysis, is restricted to refer to points $\left(x_{i}, y_{i}\right)$ that are irregularly spaced in the $x$ - and $y$-directions considered separately, but not completely irregularly in the $x, y$-plane. Consequently, interpolation formulas given for those so-called irregularly-spaced points are usually restricted to arguments $\left(x_{i}, y_{i}\right)$ that lie in a rectangular grid formed by rectangles of different sizes. For attempts to give suitable definitions of confluent forms, in connection with several suggested new divided difference formulas for functions of two variables where the arguments $\left(x_{i}, y_{i}\right)$ are spaced in a completely irregular manner, see [2] and [3].

The wide restrictions on the composition of the binary polynomials and the arrangement of the points $\left(x_{i}, y_{i}\right)$, when there does exist a solution to a bivariate osculatory or hyperosculatory interpolation problem, is indicated in the structure of

* In [1] there are many illustrations for both cases, e.g., the simplest, for the former, being the general impossibility of fitting $a+b x+c y+d x^{2}+e x y+f y^{2}$ to $f_{i}, f_{x_{i}}$ and $f_{y_{i}}, i=0,1$.

** E.g., while we may fit a binary quadratic to any $f(x, y)$ specified at the 6 points $(0,0),(1,0)$, $(0,1),(1,1),(2,1)$ and $(1,2)$, there is no limiting confluent form as $(1,0),(0,1) \rightarrow(0,0)$ and $(2,1)$, $(1,2) \rightarrow(1,1)$ (see previous footnote). 
the relevant determinant. It seems from experience that we must, in the confluent cases, avoid a binary polynomial whose general functional appearance is too symmetrical in $x$ and $y$, and a configuration of the points $\left(x_{i}, y_{i}\right)$ that is too regular. Apparently, for the confluent cases in one variable, this problem does not arise because the determinants are closely related to the nonvanishing Vandermondian. But it appears that in the confluent cases for two variables over a Cartesian grid, where the binary polynomial does not lack solutions for every configuration of the points $\left(x_{i}, y_{i}\right)$, there are still a number of ways in which the horizontal or vertical alignment of even some of the points $\left(x_{i}, y_{i}\right)$ may cause the determinant to vanish for many configurations of all the points $\left(x_{i}, y_{i}\right)$.

3. Change of Variables. Now it is convenient to shift variables from $x, y$ to $p, q$, where $p=\left(x-x_{0}\right) / h$ and $q=\left(y-y_{0}\right) / k$. Then $f(x, y)$ will be denoted by $F(p, q)$, and $f_{i} \equiv f\left(x_{i}, y_{i}\right)=F\left(p_{i}, q_{i}\right) \equiv F_{i}$. The partial derivatives of $F(p, q)$ with respect to $p$ and $q$ at $p=p_{i}$ and $q=q_{i}$, namely $F_{p_{i}} \equiv F_{p}\left(p_{i}, q_{i}\right), F_{q_{i}} \equiv F_{q}\left(p_{i}, q_{i}\right)$, $F_{p p_{i}} \equiv F_{p p}\left(p_{i}, q_{i}\right), F_{p q i} \equiv F_{p q}\left(p_{i}, q_{i}\right)$ and $F_{q q i} \equiv F_{q q}\left(p_{i}, q_{i}\right)$ are related to the partial derivatives of $f(x, y)$ with respect to $x$ and $y$ at $x=x_{i}$ and $y=y_{i}$ by $F_{p_{i}}=h f_{x_{i}}$, $F_{a i}=k f_{y i}, F_{p p_{i}}=h^{2} f_{x x_{i}}, F_{p q_{i}}=h k f_{x y i}$ and $F_{a q_{i}}=k^{2} f_{y y_{i}}$. In general,

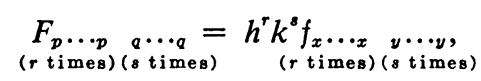

so that for small $h$ and $k$, as $r+s$ increases, the

$$
\underset{(r \text { times })(s \text { times })}{F_{p} \cdots p}
$$

becomes very much smaller than the corresponding

$$
\underset{(r \text { times })(8 \text { times })}{f}
$$

The interpolating binary nic $p_{n}(x, y)$ is denoted by $P_{n}(p, q)$.

4. Remainder Terms. To estimate the accuracy of bivariate hyperosculatory interpolation of $F(p, q)$ by a uniquely determined $P_{n}(p, q)$, in the absence of an exact expression for the remainder $F(p, q)-P_{n}(p, q)$, we obtain its dominant terms from its Taylor expansion about $\left(p_{0}, q_{0}\right)=(0,0)$, just as in [1]. One straightforward way to find those dominant terms (which is not the most convenient way in view of IV below) is to expand each

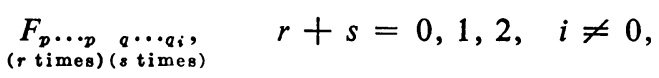

that occurs in $P_{n}(p, q)$, about $(0,0)$, and to subtract that form of $P_{n}(p, q)$ from the Taylor series for $F(p, q)$ about $(0,0)$. The remainder is then seen to be given by

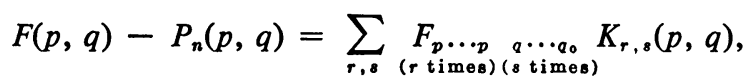

where $K_{r, e}(p, q)$ are polynomials in $p$ and $q$. From the uniqueness of both $P_{n}(p, q)$ and the Taylor series, we obtain immediately the following four guiding rules as to which $(r, s)$ terms are to be found in the right member of (1), and also how to find the polynomials $K_{r, s}(p, q)$ most conveniently: 
I. If $P_{n}(p, q)$ contains a term $K p^{r} q^{s}$, there will be no $(r, s)$ term in the right member of (1).

II. If $P_{n}(p, q)$ lacks a term $K p^{r} q^{s}$, there will be an $(r, s)$ term in the right member of (1).

III. If $P_{n}(p, q)$ has a term of the form $K\left(p^{r} q^{s}+p^{s} q^{r}\right)$, then in (1), $K_{\mathrm{e}, \mathrm{r}}(p, q)=$ $-K_{r, 8}(p, q)$.

IV. The application of (1) to $F(p, q)=p^{r} q^{s}$, for which the right member has just a single term, yields for $K_{r, s}(p, q)$ the explicit formula

$$
K_{r, s}(p, q)=(1 / r ! s !)\left[p^{r} q^{q}-P_{n}(p, q) \text { for the function } p^{r} q^{s}\right] \text {. }
$$

5. Determination of Formulas. All formulas have the same quadratic part, the first six terms of the Taylor series about $\left(p_{0}, q_{0}\right)=(0,0)$, namely,

$$
F(p, q)=F_{0}+p F_{p_{0}}+q F_{a_{0}}+\frac{p^{2}}{2} F_{p p_{0}}+p q F_{p q_{0}}+\frac{q^{2}}{2} F_{a a_{0}}+\cdots .
$$

Those six terms also constitute the optimal hyperosculatory formula for $m=1$.

For $m=2$, say $(0,0)$ and $\left(p_{1}, q_{1}\right)$, we must solve a $6 \times 6$ linear system for the coefficients of the higher degree terms in $P_{n}(p, q)$, to meet the interpolation conditions at $\left(p_{1}, q_{1}\right)$. In view of I-III above, those six terms should be of the lowest possible degree. Consider the $6 \times 9$ matrix of $p^{i} q^{i}$ with its first two partial derivatives, $i+j=3$ and 4 , for every $\left(p_{1}, q_{1}\right)$. We may drop the subscript 1 and refer to that matrix as $P$. No polynomial $P_{n}(p, q)$ can have four independent cubic terms because the four cubic columns in $P$ are linearly dependent, as seen from the multipliers 1 , $-3 p / q, 3 p^{2} / q^{2}$ and $-p^{3} / q^{3}$ for $q \neq 0$, and a vanishing $q^{3}$ column for $q=0$. The author conjectured, and T. N. E. Greville proved in 1960, that rank $P<6$. Greville showed the linear dependence of the rows of $P$ by verifying that for any polynomial $P(p, q)$ consisting only of cubic and quartic terms,

$$
P(p, q) \equiv \frac{1}{2} p P_{p}+\frac{1}{2} q P_{q}-\frac{1}{12} p^{2} P_{p p}-\frac{1}{6} p q P_{p q}-\frac{1}{12} q^{2} P_{q q} \cdot * * *
$$

These properties of $P$ show that no two-point formula can have more than five independent cubic and quartic terms, of which there cannot be more than three cubic terms. Thus an optimal formula, from the standpoint of highest degree dominant $h^{r} k^{s}$ terms in the remainder, should have three cubic, two quartic and one quintic terms. The closest configuration of two points is $\left(p_{0}, q_{0}\right)=(0,0)$ and $\left(p_{1}, q_{1}\right)=(1,0) . \dagger$ It has the drawback of not being symmetrical with respect to the $p, q$-grid. At this point, we introduce the notation " $\Delta$ " for the determinant of the linear system of equations for the coefficients in any formula under consideration throughout this article. For $(0,0)$ and $(1,0)$, the nonquadratic part of the interpolating quintic $P_{5}(p, q)$, subject to the conditions of $\Delta \neq 0$ and $P_{5}(p, q)$ being symmetrical in $p$ and $q$, can be only of the form $a\left(p^{3}+q^{3}\right)+b p^{2} q+c p q^{2}+d\left(p^{4}+q^{4}\right)+e\left(p^{3} q+p q^{3}\right)$

\footnotetext{
*** The result in [1] on the impossibility of finding a binary quadratic for two-point osculatory interpolation is expressible as the simplest Greville-type identity: If $P(p, q)$ has only quadratic terms, $P(p, q) \equiv \frac{1}{2} p P_{p}+\frac{1}{2} q P_{q}$.

$\dagger$ For $(0,0)$ and $(0,1)$ just interchange $p$ and $q$ in the formula for $(0,0)$ and $(1,0)$.

$\ddagger$ Here symmetrical means not unchanged in value on interchanging $p$ and $q \downarrow$ but unchanged in general functional form.
} 
$+f\left(p^{5}+q^{5}\right)$. For the corresponding formula, with remainder terms through the 5th order, see AI in Section 6 below. The points $\left(p_{0}, q_{0}\right)=(0,0)$ and $\left(p_{1}, q_{1}\right)=$ $(1,1)$ are situated symmetrically with respect to the $p, q$-grid, though their distance apart is nearly $1 \frac{1}{2}$ times that for $(0,0)$ and $(1,0)$. There are altogether 30 symmetrical forms for $P_{5}(p, q)$, of which 18 have $\Delta=0$ and 12 have $\Delta \neq 0$ for that configuration. Of the permissible 12, two representative choices of $P_{s}(p, q)$ are $P_{5}(p, q)=$ quadratic part $+a p^{3}+b\left(p^{2} q+p q^{2}\right)+c q^{3}+d p^{4}+e q^{4}+f\left(p^{5}+q^{5}\right)$ ("extreme" power weighted), and $P_{5}(p, q)=$ quadratic part $+a\left(p^{3}+q^{3}\right)+b p^{2} q+c p q^{2}+$ $d p^{3} q+e p q^{3}+f\left(p^{3} q^{2}+p^{2} q^{3}\right)$ ("central" power weighted). For the corresponding formulas, with remainder terms through the 5th order, see AII and AIII in Section 6 below.

For $m=3$, say for points $(0,0),\left(p_{1}, q_{1}\right)$ and $\left(p_{2}, q_{2}\right)$, it is natural to meet the 18 interpolation conditions with a polynomial $P_{s}(p, q)$ having the 15 terms of the complete quartic and which preserves its symmetrical form with the three quintic terms $a\left(p^{5}+q^{5}\right)+b\left(p^{4} q+p q^{4}\right)+c\left(p^{3} q^{2}+p^{2} q^{3}\right)$. Obtaining $P_{5}(p, q)$ requires the solution of a $12 \times 12$ linear system. It was found that $\Delta=0$ for these 14 configurations of base points (heavier dot for $(0,0)$ ):

and $\Delta \neq 0$ for these four configurations:

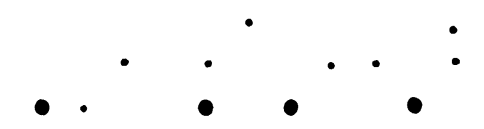

The scarcity of configurations where $\Delta \neq 0$ is underscored by noting that the last three are variations of just

under one of or both the transformations $(p, q) \rightarrow(q, p)$ and $(p, q) \rightarrow(2-p, 1-q)$ which leave the above selected $P_{s}(p, q)$ unchanged in its symmetrical form. For the formula corresponding to the configuration

with remainder terms through the 6th order, see BI in Section 6 below. We may use BI for any other "knight's move" configuration of three points, with the proper definition of $p$ and $q$. E.g., for $(0,1),(1,1)$ and $(2,0)$ replace $(p, q)$ by $(p, 1-q)$, remembering not to overlook the changes of sign in

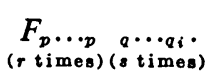

A different nonsymmetrical $P_{5}(p, q)$ was employed for the very nice-looking symmetrical configuration 
having the least diameter of $2^{1 / 2}=1.41 \ldots$ (cf. with diameter of $5^{1 / 2}=2.24 \ldots$ for

Of the 20 possible combinations of three terms in $p^{i} q^{i}, i+j=5$, to add to the complete quartic part of $P_{5}(p, q), \Delta \neq 0$ for just these $4: p^{5}, p^{4} q, q^{5} ; p^{5}, p q^{4}, q^{5} ; p^{5}$, $p^{3} q^{2}, q^{5} ; p^{5}, p^{2} q^{3}, q^{5}$. The second and fourth combinations are essentially the same as the first and third resp. because of the symmetrical form of both the

configuration and the complete quartic part of $P_{5}(p, q)$. The $p^{5}, p^{3} q^{2}, q^{5}$ combination was chosen because it appears more balanced in $p$ and $q$ than $p^{5}, p^{4} q, q^{5}$. For the corresponding formula, with remainder terms through the 6 th order, see BII in Section 6 below. As a rule, BII is preferable to BI because it has fewer terms, smaller coefficients, a generally smaller remainder, $\dagger^{\dagger} \dagger$ is easier to compute, and the

-

-

configuration is more convenient and adaptable than

(e.g., in interpolating for the solution to a problem in a region that is bounded naturally by a square, where the

configuration might be inapplicable or less convenient).

For $m=4$, for points $(0,0),\left(p_{1}, q_{1}\right),\left(p_{2}, q_{2}\right)$ and $\left(p_{3}, q_{3}\right)$, there is the very natural and attractive-looking

configuration. The 24 interpolation conditions are satisfied by a symmetrical sextic $P_{6}(p, q)$ that has the 21 terms of a complete binary quintic and three terms in $p^{i} q^{i}, i+$ $j=6$, which must include $p^{3} q^{3}$ to have symmetry. Of the combinations $p^{6}$, $q^{6}$, or $p^{5} q, p q^{5}$, or $p^{4} q^{2}, p^{2} q^{4}, \Delta \neq 0$ only for $p^{5} q, p q^{5}$. For the corresponding formula, with remainder terms through the 7th order, see CI in Section 6 below.

To go beyond $m=4$ for second derivative formulas, or to find formulas for $m \geqq 2$ involving third- or higher-order derivatives, it is recommended that one develop

t†† Exceptions may occur when

$$
\begin{gathered}
F_{p \cdots p} q \cdots q_{0} \\
(\text { times) }(\bullet \text { times })
\end{gathered}
$$

is practically the same as

$$
\underset{(s \text { times })(r \text { times })}{F_{p \cdots p} \cdots q_{0},} \quad r+s=5
$$

for $h$ and $k$ sufficiently small. 
a single comprehensive machine program for eliminating $P_{n}(p, q)$ and configurations of $\left(p_{i}, q_{i}\right)$ where $\Delta=0$, and then solving for the coefficients of $P_{n}(p, q)$ and the dominant remainder terms in cases where $\Delta \neq 0$. It may be anticipated, on the basis of [1] and this present work, that one will discover an even greater scarcity of satisfactory interpolating polynomials $P_{n}(p, q)$ and sufficiently close configurations of points $\left(p_{i}, q_{i}\right)$ for which $\Delta \neq 0$. For instance, in two-point hyperosculatory interpolation involving third derivatives, we might wish to satisfy the 10 interpolation conditions at $\left(p_{1}, q_{1}\right)$ with a $P_{5}(p, q)$ having, beyond a complete cubic part for the 10 conditions at $(0,0), 10$ of the 11 possible quartic and quintic terms $p^{i} q^{i}, i+j=4$ and 5. But that is impossible because rank $R<10$, where $R$ is the $10 \times 11$ matrix of $p^{i} q^{i}$ and its first three partial derivatives. In fact, the rows of $R$ satisfy a very strong Greville-type identity, since the first six alone are linearly dependent, the multipliers being $1,-2 p / 5,-2 q / 5, p^{2} / 20, p q / 10$ and $q^{2} / 20$. This implies that if we replace the four third derivative conditions at $\left(p_{1}, q_{1}\right)$ by any other four conditions, not necessarily involving derivatives, or even the point $\left(p_{1}, q_{1}\right)$, the corresponding $\Delta$ will still vanish.

6. Schedule of Formulas. Every formula is given in matrix form

$$
F(p, q)=\alpha A \beta^{T}+\gamma B \delta^{T}+\cdots,
$$

where $\alpha A \beta^{T}$ is the interpolating polynomial $P_{n}(p, q)$ and $\gamma B \delta^{T}$ is the sum of the dominant remainder terms. The matrix form has the advantage (besides its obvious compactness and convenience) of providing either the coefficients $a, b, c, \ldots$ etc., by first taking the product $\alpha A$, or the polynomial coefficients of

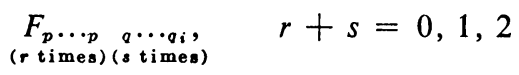

(analogous to Lagrange interpolation coefficients), by first taking the product $A \beta^{T}$. Obtaining $\alpha A\left(A \beta^{T}\right)$ first might be preferable for machine storage when there are fewer (many) sets of

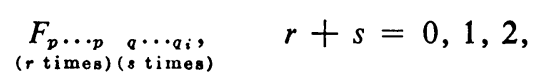

and many (fewer) arguments $p$ and $q$.

Every formula given below, including all the dominant remainder terms, was checked by having it reproduce exactly a function $F(p, q)$ that was chosen to be a polynomial with integral coefficients and of such degree that the dominant remainder terms constituted the entire remainder. 
Two-Point Formulas.

AI. $\left(p_{0}, q_{0}\right)=(0,0),\left(p_{1}, q_{1}\right)=(1,0)$.

$\alpha$ is the $1 \times 12$ matrix $\left\|F_{0}, F_{p_{0}}, F_{a_{0}}, F_{p p_{0}}, F_{p a_{0}}, F_{a a_{0}}, F_{1}, F_{p_{1}}, F_{a_{1}}, F_{p p_{1}}, F_{p q_{1}}, F_{a q_{1}}\right\|$, $A$ is the $12 \times 12$ matrix

$$
\left\|\begin{array}{rrrrrrrrrrrr}
1 & 0 & 0 & 0 & 0 & 0 & -10 & 0 & 0 & 15 & 0 & -6 \\
0 & 1 & 0 & 0 & 0 & 0 & -6 & 0 & 0 & 8 & 0 & -5 \\
0 & 0 & 1 & 0 & 0 & 0 & 0 & -3 & 0 & 0 & 2 & 0 \\
0 & 0 & 0 & \frac{1}{2} & 0 & 0 & -\frac{3}{2} & 0 & 0 & \frac{5}{2} & 0 & -\frac{1}{2} \\
0 & 0 & 0 & 0 & 1 & 0 & 0 & -2 & 0 & 0 & 1 & 0 \\
0 & 0 & 0 & 0 & 0 & \frac{1}{2} & 0 & 0 & -\frac{1}{2} & 0 & 0 & 0 \\
0 & 0 & 0 & 0 & 0 & 0 & 10 & 0 & 0 & -16 & 0 & 6 \\
0 & 0 & 0 & 0 & 0 & 0 & -4 & 0 & 0 & 7 & 0 & -5 \\
0 & 0 & 0 & 0 & 0 & 0 & 0 & 3 & 0 & 0 & -2 & 0 \\
0 & 0 & 0 & 0 & 0 & 0 & \frac{1}{2} & 0 & 0 & -1 & 0 & \frac{1}{2} \\
0 & 0 & 0 & 0 & 0 & 0 & 0 & -1 & 0 & 0 & 1 & 0 \\
0 & 0 & 0 & 0 & 0 & 0 & 0 & 0 & \frac{1}{2} & 0 & 0 & 0
\end{array}\right\|
$$

$\beta$ is the $1 \times 12$ matrix $\| 1, p, q, p^{2}, p q, q^{2}, p^{3}+q^{3}, p^{2} q, p q^{2}, p^{4}+q^{4}, p^{3} q+p q^{3}$, $p^{5}+q^{5} \|$,

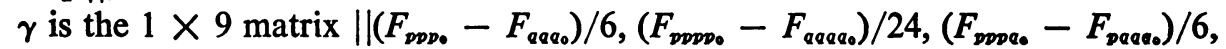

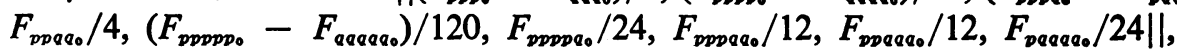

$B$ is the $9 \times 12$ matrix

$$
\left\|\begin{array}{cccccccccccc}
0 & 0 & -1 & 0 & 0 & 0 & 0 & 0 & 0 & 0 & 0 & 0 \\
0 & 0 & 0 & 0 & 0 & 0 & -1 & 0 & 0 & 0 & 0 & 0 \\
0 & 0 & 0 & 0 & 0 & -1 & 0 & 0 & 0 & 0 & 0 & 0 \\
0 & -1 & 0 & 0 & 1 & 0 & 0 & 0 & 0 & 0 & 0 & 0 \\
0 & 0 & 0 & 0 & 0 & 0 & 0 & 0 & 0 & 0 & 0 & -1 \\
1 & 0 & 0 & -2 & 0 & -2 & 0 & 1 & 0 & 0 & 0 & 0 \\
0 & -1 & 0 & 0 & 0 & 0 & 0 & 0 & 1 & 0 & 0 & 0 \\
0 & 0 & 0 & 0 & 0 & 0 & 0 & 0 & 0 & 1 & 0 & 0 \\
0 & 0 & 0 & 0 & 0 & 0 & 0 & 0 & 0 & 0 & 1 & 0
\end{array}\right\|
$$

and $\delta$ is the $1 \times 12$ matrix $\left\|p^{2} q, p q^{2}, q^{3}, p^{3} q, p^{2} q^{2}, p q^{3}, q^{4}, p^{4} q, p^{3} q^{2}, p^{2} q^{3}, p q^{4}, q^{5}\right\|$.

AII. $\left(p_{0}, q_{0}\right)=(0,0),\left(p_{1}, q_{1}\right)=(1,1)$.

$\alpha$ has the same form as in AI above, but a different value, since $\left(p_{1}, q_{1}\right)=(1,1)$ instead of $(1,0)$. 
$A$ is the $12 \times 12$ matrix

$$
\left\|\begin{array}{rrrrrrrrrrrr}
1 & 0 & 0 & 0 & 0 & 0 & -5 & 0 & -5 & \frac{15}{2} & \frac{15}{2} & -3 \\
0 & 1 & 0 & 0 & 0 & 0 & -\frac{7}{2} & 0 & -\frac{5}{2} & \frac{17}{4} & \frac{15}{4} & -\frac{5}{2} \\
0 & 0 & 1 & 0 & 0 & 0 & -\frac{5}{2} & 0 & -\frac{7}{2} & \frac{15}{4} & \frac{17}{4} & -\frac{5}{2} \\
0 & 0 & 0 & \frac{1}{2} & 0 & 0 & -\frac{15}{12} & 0 & -\frac{5}{12} & \frac{7}{8} & \frac{5}{8} & -\frac{1}{4} \\
0 & 0 & 0 & 0 & 1 & 0 & -\frac{5}{4} & -\frac{1}{4} & -\frac{5}{4} & \frac{3}{2} & \frac{5}{2} & -\frac{1}{2} \\
0 & 0 & 0 & 0 & 0 & \frac{1}{2} & -\frac{5}{12} & 0 & -\frac{15}{12} & \frac{5}{8} & \frac{7}{8} & -\frac{1}{4} \\
0 & 0 & 0 & 0 & 0 & 0 & 0 & 0 & 5 & -\frac{15}{2} & -\frac{15}{2} & 3 \\
0 & 0 & 0 & 0 & 0 & 0 & -\frac{5}{2} & 0 & -\frac{5}{2} & \frac{15}{4} & \frac{15}{4} & -\frac{5}{2} \\
0 & 0 & 0 & 0 & 0 & 0 & -\frac{5}{2} & 0 & -\frac{5}{2} & \frac{15}{4} & \frac{15}{4} & -\frac{5}{2} \\
0 & 0 & 0 & 0 & 0 & 0 & \frac{1}{12} & 0 & \frac{5}{12} & -\frac{3}{8} & -\frac{5}{8} & \frac{1}{4} \\
0 & 0 & 0 & 0 & 0 & 0 & \frac{1}{4} & \frac{1}{4} & \frac{1}{4} & -1 & -1 & \frac{1}{2} \\
0 & 0 & 0 & 0 & 0 & 0 & \frac{5}{12} & 0 & \frac{1}{12} & -\frac{5}{8} & -\frac{5}{8} & \frac{1}{4}
\end{array}\right\|
$$

$\beta$ is the $1 \times 12$ matrix $\left\|1, p, q, p^{2}, p q, q^{2}, p^{3}, p^{2} q+p q^{2}, q^{3}, p^{4}, q^{4}, p^{5}+q^{5}\right\|$, $\gamma$ is the $1 \times 9$ matrix $\|\left(F_{p p q_{0}}-F_{p q q_{0}}\right) / 2, F_{p p p q_{0}} / 6, F_{p p q q_{0}} / 4, F_{p q q e_{0}} / 6$, $\left(F_{\text {pppppo }}-F_{\text {qeqeq }}\right) / 120, F_{\text {ppppq }} / 24, F_{\text {pppeqo }} / 12, F_{\text {ppqqa }} / 12, F_{\text {pqqeq }} / 24 \|$,

$B$ is the $9 \times 15$ matrix

$$
\mid \begin{array}{ccccccccccccccc}
-\frac{1}{6} & \frac{1}{2} & -\frac{1}{2} & \frac{1}{6} & 0 & 0 & 0 & 0 & 0 & 0 & 0 & 0 & 0 & 0 & 0 \\
\frac{3}{4} & -\frac{3}{4} & -\frac{3}{4} & \frac{5}{4} & -\frac{3}{4} & 1 & 0 & 0 & -\frac{1}{4} & 0 & 0 & 0 & 0 & 0 & 0 \\
1 & -1 & -1 & 1 & -\frac{1}{2} & 0 & 1 & 0 & -\frac{1}{2} & 0 & 0 & 0 & 0 & 0 & 0 \\
\frac{5}{4} & -\frac{3}{4} & -\frac{5}{4} & \frac{3}{4} & -\frac{1}{4} & 0 & 0 & 1 & -\frac{3}{4} & 0 & 0 & 0 & 0 & 0 & 0 \\
\frac{5}{6} & 0 & 0 & -\frac{5}{6} & -\frac{5}{4} & 0 & 0 & 0 & \frac{5}{4} & \frac{1}{2} & 0 & 0 & 0 & 0 & -\frac{1}{2} \\
\frac{3}{2} & -1 & -1 & \frac{1}{2} & -\frac{3}{4} & 0 & 0 & 0 & \frac{3}{4} & -\frac{1}{2} & 1 & 0 & 0 & 0 & -\frac{1}{2} \\
\frac{5}{5} & -\frac{5}{2} & -\frac{5}{2} & \frac{4}{3} & -\frac{1}{4} & 0 & 0 & 0 & \frac{1}{4} & -\frac{1}{2} & 0 & 1 & 0 & 0 & -\frac{1}{2} \\
\frac{4}{5} & -\frac{3}{2} & -\frac{3}{2} & \frac{5}{3} & \frac{1}{4} & 0 & 0 & 0 & -\frac{1}{4} & -\frac{1}{2} & 0 & 0 & 1 & 0 & -\frac{1}{2} \\
\frac{1}{2} & -1 & -1 & \frac{5}{2} & \frac{3}{4} & 0 & 0 & 0 & -\frac{3}{4} & -\frac{1}{2} & 0 & 0 & 0 & 1 & -\frac{1}{2}
\end{array} \|
$$

and $\delta$ is the $1 \times 15$ matrix $\| p^{3}, p^{2} q, p q^{2}, q^{3}, p^{4}, p^{3} q, p^{2} q^{2}, p q^{3}, q^{4}, p^{8}, p^{4} q, p^{3} q^{2}, p^{2} q^{3}$, $p q^{4}, q^{8} \|$.

AIII. $\left(p_{0}, q_{0}\right)=(0,0),\left(p_{1}, q_{1}\right)=(1,1)$.

$\alpha$ is identical with that in AII. 
$A$ is the $12 \times 12$ matrix

$$
\begin{array}{||llllllrrrrrr}
1 & 0 & 0 & 0 & 0 & 0 & -\frac{11}{4} & -\frac{9}{4} & -\frac{9}{4} & \frac{15}{2} & \frac{15}{2} & -3 \\
0 & 1 & 0 & 0 & 0 & 0 & -\frac{3}{2} & -3 & 0 & \frac{9}{2} & \frac{7}{2} & -\frac{3}{2} \\
0 & 0 & 1 & 0 & 0 & 0 & -\frac{3}{2} & 0 & -3 & \frac{7}{2} & \frac{9}{2} & -\frac{3}{2} \\
0 & 0 & 0 & \frac{1}{2} & 0 & 0 & -\frac{3}{8} & -\frac{11}{8} & \frac{5}{8} & 1 & \frac{1}{2} & -\frac{1}{4} \\
0 & 0 & 0 & 0 & 1 & 0 & -\frac{1}{2} & -1 & -1 & \frac{3}{2} & \frac{3}{2} & -\frac{1}{2} \\
0 & 0 & 0 & 0 & 0 & \frac{1}{2} & -\frac{3}{8} & \frac{5}{8} & -\frac{11}{8} & \frac{1}{2} & 1 & -\frac{1}{4} \\
0 & 0 & 0 & 0 & 0 & 0 & \frac{11}{4} & \frac{9}{4} & \frac{9}{4} & -\frac{15}{2} & -\frac{15}{2} & 3 \\
0 & 0 & 0 & 0 & 0 & 0 & -\frac{5}{4} & \frac{3}{4} & -\frac{9}{4} & 3 & 4 & -\frac{3}{2} \\
0 & 0 & 0 & 0 & 0 & 0 & -\frac{5}{4} & -\frac{9}{4} & \frac{3}{4} & 4 & 3 & -\frac{3}{2} \\
0 & 0 & 0 & 0 & 0 & 0 & \frac{1}{4} & -\frac{1}{2} & \frac{1}{2} & -\frac{1}{4} & -\frac{3}{4} & \frac{1}{4} \\
0 & 0 & 0 & 0 & 0 & 0 & \frac{1}{4} & \frac{1}{4} & \frac{1}{4} & -1 & -1 & \frac{1}{2} \\
0 & 0 & 0 & 0 & 0 & 0 & \frac{1}{4} & \frac{1}{2} & -\frac{1}{2} & -\frac{3}{4} & -\frac{1}{4} & \frac{1}{4}
\end{array} \|
$$

$\beta$ is the $1 \times 12$ matrix $\left\|1, p, q, p^{2}, p q, q^{2}, p^{3}+q^{3}, p^{2} q, p q^{2}, p^{3} q, p q^{3}, p^{3} q^{2}+p^{2} q^{3}\right\|$, $\gamma$ is the $1 \times 9$ matrix $\|\left(F_{p p_{0}}-F_{q q q_{0}}\right) / 6, F_{p p p_{0} p_{0}} / 24, F_{p q_{q u}} / 4, F_{q q q q_{0}} / 24, F_{p p p p p_{0}} / 120$, $F_{\text {ppppa }} / 24,\left(F_{\text {pppqa }}-F_{\text {ppqaq }}\right) / 12, F_{\text {pqeqa }} / 24, F_{\text {qqqaq }} / 120 \|$,

$B$ is the $9 \times 15$ matrix

$$
\begin{array}{||ccccccccccccccc}
\frac{1}{2} & -\frac{3}{2} & \frac{3}{2} & -\frac{1}{2} & 0 & 0 & 0 & 0 & 0 & 0 & 0 & 0 & 0 & 0 & 0 \\
-\frac{3}{4} & \frac{3}{4} & \frac{3}{4} & -\frac{3}{4} & 1 & -\frac{3}{2} & 0 & \frac{1}{2} & 0 & 0 & 0 & 0 & 0 & 0 & 0 \\
\frac{1}{4} & -\frac{1}{4} & -\frac{1}{4} & \frac{1}{4} & 0 & -\frac{1}{2} & 1 & -\frac{1}{2} & 0 & 0 & 0 & 0 & 0 & 0 & 0 \\
-\frac{3}{4} & \frac{5}{4} & \frac{3}{4} & -\frac{3}{4} & 0 & \frac{1}{2} & 0 & -\frac{3}{2} & 1 & 0 & 0 & 0 & 0 & 0 & 0 \\
-\frac{3}{2} & 4 & -1 & -\frac{3}{2} & 0 & -\frac{5}{2} & 0 & \frac{5}{2} & 0 & 1 & 0 & -\frac{1}{2} & -\frac{1}{2} & 0 & 0 \\
-\frac{1}{2} & 2 & -1 & -\frac{1}{2} & 0 & -\frac{3}{2} & 0 & \frac{3}{2} & 0 & 0 & 1 & -\frac{1}{2} & -\frac{1}{2} & 0 & 0 \\
0 & \frac{1}{2} & -\frac{1}{2} & 0 & 0 & -\frac{1}{2} & 0 & \frac{1}{2} & 0 & 0 & 0 & \frac{1}{2} & -\frac{1}{2} & 0 & 0 \\
-\frac{1}{2} & -1 & 2 & -\frac{1}{2} & 0 & \frac{3}{2} & 0 & -\frac{3}{2} & 0 & 0 & 0 & -\frac{1}{2} & -\frac{1}{2} & 1 & 0 \\
-\frac{3}{2} & -1 & 4 & -\frac{3}{2} & 0 & \frac{5}{2} & 0 & -\frac{5}{2} & 0 & 0 & 0 & -\frac{1}{2} & -\frac{1}{2} & 0 & 1
\end{array} \|
$$

and $\delta$ is identical with that in AII. 
Three-Point Formulas.

BI. $\left(p_{0}, q_{0}\right)=(0,0),\left(p_{1}, q_{1}\right)=(1,0),\left(p_{2}, q_{2}\right)=(2,1)$. $\alpha$ is the $1 \times 18$ matrix $\| F_{0}, F_{p_{0}}, F_{q_{0}}, F_{p p_{0}}, F_{p q_{0}}, F_{a q_{0}}, F_{1}, F_{p_{1}}, F_{q_{1}}, F_{p p_{1}}, F_{p q_{1}}, F_{a q_{1}}$, $F_{2}, F_{p_{2}}, F_{a_{2}}, F_{p p_{1}}, F_{p a_{2}}, F_{a a_{1}} \|$,

$A$ is the $18 \times 18$ matrix

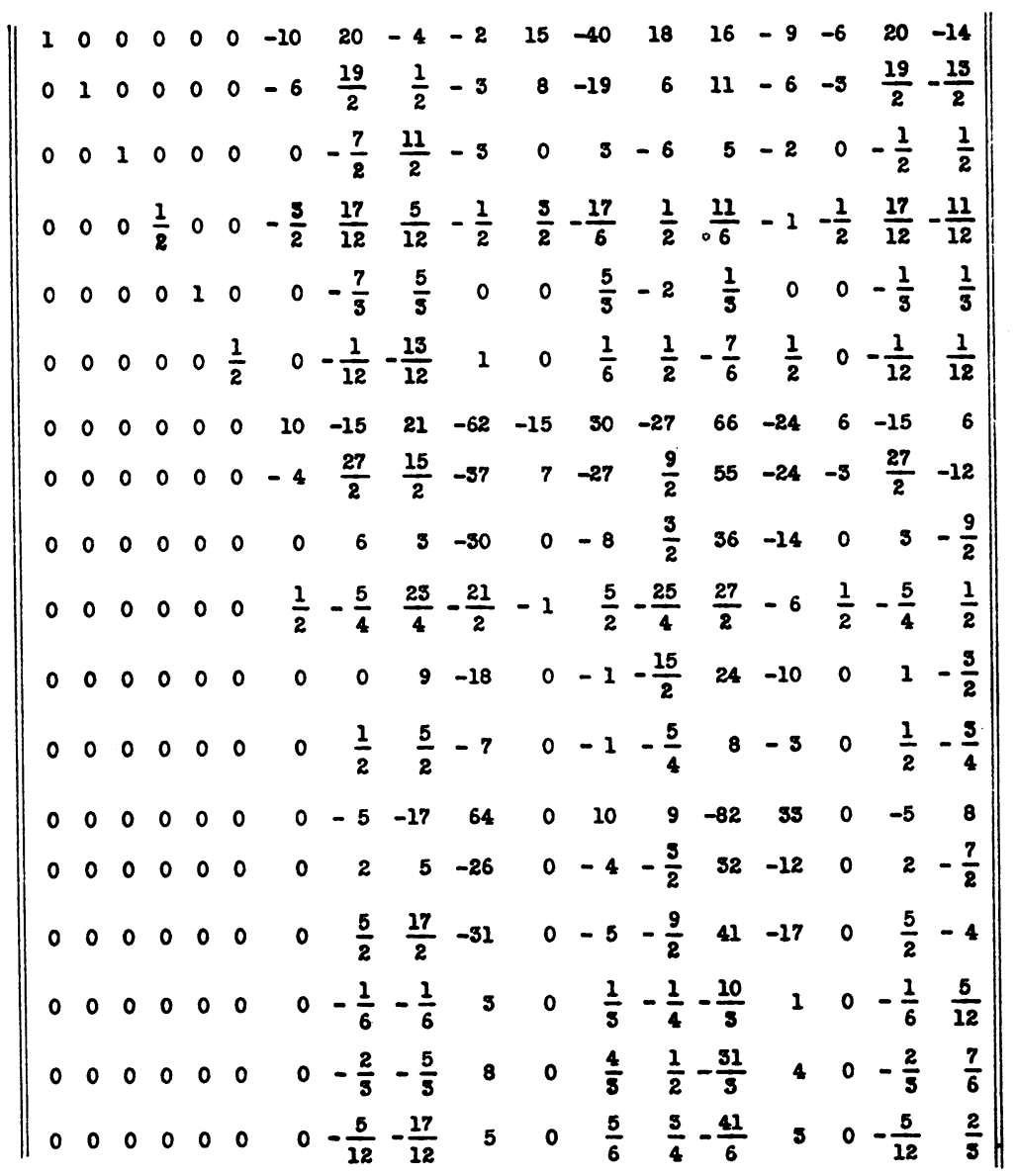

$\beta$ is the $1 \times 18$ matrix $\| 1, p, q, p^{2}, p q, q^{2}, p^{3}, p^{2} q, p q^{2}, q^{3}, p^{4}, p^{3} q, p^{2} q^{2}, p q^{3}, q^{4}, p^{5}+$ $q^{5}, p^{4} q+p q^{4}, p^{3} q^{2}+p^{2} q^{3} \|$

$\gamma$ is the $1 \times 10$ matrix $\|\left(F_{p p p p p_{0}}-F_{\text {qqQqQ }}\right) / 120,\left(F_{p p p p q 0}-F_{\text {pqqQQ }}\right) / 24$,

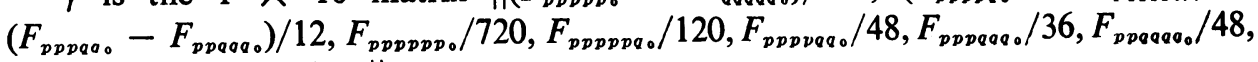
$F_{\text {pqaqa o }} / 120, F_{\text {qeqaqe }} / 720 \|$, 
$B$ is the $10 \times 22$ matrix

$$
\begin{array}{||ccccccccccccccccccccccc||}
0 & -\frac{5}{6} & -\frac{17}{6} & 9 & 0 & \frac{5}{3} & \frac{3}{2} & -\frac{41}{3} & 8 & 0 & -\frac{5}{6} & \frac{4}{3} & \frac{4}{3} & -\frac{5}{6} & -1 & 0 & 0 & 0 & 0 & 0 & 0 & 0 \\
0 & -\frac{2}{3} & -\frac{5}{3} & 6 & 0 & \frac{4}{3} & \frac{1}{2} & -\frac{28}{3} & 6 & 0 & -\frac{2}{3} & \frac{7}{6} & \frac{7}{6} & -\frac{5}{3} & 0 & 0 & 0 & 0 & 0 & 0 & 0 & 0 \\
0 & -\frac{1}{3} & -\frac{1}{3} & 2 & 0 & \frac{2}{3} & -\frac{1}{2} & -\frac{8}{3} & 2 & 0 & -\frac{1}{3} & \frac{5}{6} & -\frac{1}{6} & -\frac{1}{3} & 0 & 0 & 0 & 0 & 0 & 0 & 0 & 0 \\
-1 & -\frac{25}{2} & -\frac{61}{2} & 55 & 5 & 25 & \frac{39}{2} & -97 & 60 & -3 & -\frac{25}{2} & 11 & 11 & -\frac{25}{2} & -3 & 1 & 0 & 0 & 0 & 0 & 0 & 0 \\
0 & -6 & -16 & 24 & 0 & 13 & 12 & -44 & 32 & 0 & -8 & 4 & 4 & -8 & 0 & 0 & 1 & 0 & 0 & 0 & 0 & 0 \\
0 & -1 & -5 & -2 & 0 & 2 & \frac{13}{2} & 0 & 6 & 0 & -1 & -\frac{5}{2} & -\frac{5}{2} & -1 & 0 & 0 & 0 & 1 & 0 & 0 & 0 & 0 \\
0 & 2 & 2 & -20 & 0 & -4 & 5 & 28 & -12 & 0 & 2 & -5 & -5 & 2 & 0 & 0 & 0 & 0 & 1 & 0 & 0 & 0 \\
0 & 5 & 7 & -50 & 0 & -6 & -\frac{3}{2} & 44 & -22 & 0 & 3 & -\frac{11}{2} & -\frac{11}{2} & 5 & 0 & 0 & 0 & 0 & 0 & 1 & 0 & 0 \\
0 & 3 & 9 & -52 & 0 & -6 & -4 & 47 & -24 & 0 & 5 & -5 & -5 & 5 & 0 & 0 & 0 & 0 & 0 & 0 & 1 & 0 \\
0 & \frac{5}{2} & \frac{17}{2} & -28 & 0 & -5 & -\frac{9}{2} & 41 & -21 & 0 & \frac{5}{2} & -4 & -4 & \frac{5}{2} & 0 & 0 & 0 & 0 & 0 & 0 & 0 & 1
\end{array} \|
$$

and $\delta$ is the $1 \times 22$ matrix $\| p^{3}, p^{2} q, p q^{2}, q^{3}, p^{4}, p^{8} q, p^{2} q^{2}, p q^{8}, q^{4}, p^{8}, p^{4} q, p^{8} q^{2}, p^{2} q^{3}$, $p q^{4}, q^{5}, p^{6}, p^{5} q, p^{4} q^{2}, p^{3} q^{3}, p^{2} q^{4}, p q^{5}, q^{6} \|$.

BII. $\left(p_{0}, q_{0}\right)=(0,0),\left(p_{1}, q_{1}\right)=(1,0),\left(p_{2}, q_{2}\right)=(0,1)$.

$\alpha$ has the same form as in BI above, but a different value, since $\left(p_{2}, q_{2}\right)=(0,1)$ instead of $(2,1)$.

$A$ is the $18 \times 18$ matrix

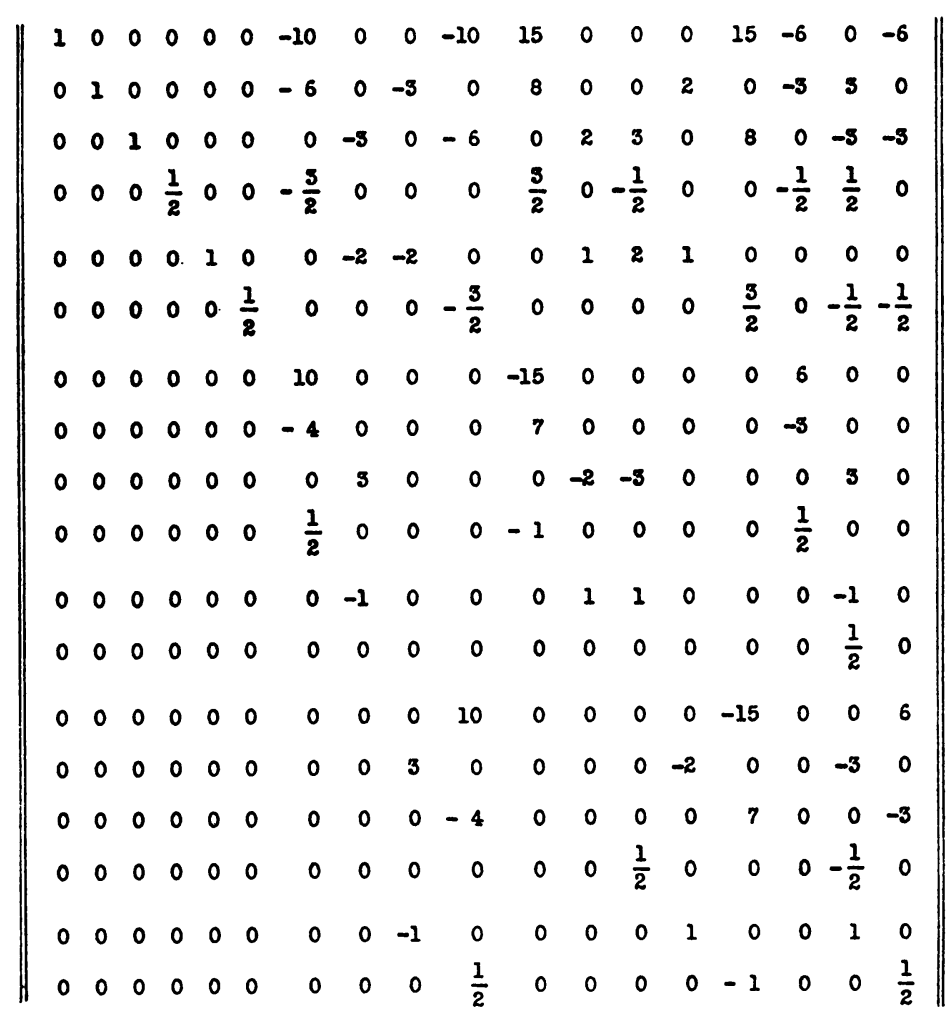


$\beta$ is the $1 \times 18$ matrix $\| 1, p, q, p^{2}, p q, q^{2}, p^{3}, p^{2} q, p q^{2}, q^{8}, p^{4}, p^{3} q, p^{2} q^{2}, p q^{3}, q^{4}, p^{5}$, $p^{8} q^{2}, q^{5} \|$,

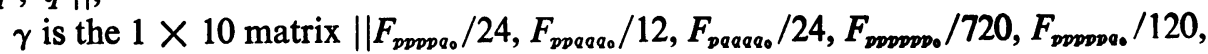

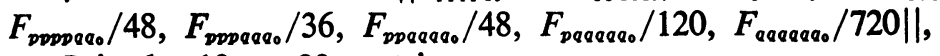

$B$ is the $10 \times 22$ matrix

$$
\begin{array}{||ccccccccccccccccccccccc||}
0 & 1 & 0 & 0 & 0 & -2 & -1 & 0 & 0 & 0 & 1 & 1 & 0 & 0 & 0 & 0 & 0 & 0 & 0 & 0 & 0 & 0 \\
0 & 0 & 0 & 0 & 0 & 0 & -1 & 0 & 0 & 0 & 0 & 1 & 1 & 0 & 0 & 0 & 0 & 0 & 0 & 0 & 0 & 0 \\
0 & 0 & 1 & 0 & 0 & 0 & 0 & -2 & 0 & 0 & 0 & -1 & 0 & 1 & 0 & 0 & 0 & 0 & 0 & 0 & 0 & 0 \\
-1 & 0 & 0 & 0 & 3 & 0 & 0 & 0 & 0 & -3 & 0 & 0 & 0 & 0 & 0 & 1 & 0 & 0 & 0 & 0 & 0 & 0 \\
0 & 2 & 0 & 0 & 0 & -3 & -2 & 0 & 0 & 0 & 0 & 2 & 0 & 0 & 0 & 0 & 1 & 0 & 0 & 0 & 0 & 0 \\
0 & 0 & 0 & 0 & 0 & 0 & 0 & 0 & 0 & 0 & 0 & -1 & 0 & 0 & 0 & 0 & 0 & 1 & 0 & 0 & 0 & 0 \\
0 & 0 & 0 & 0 & 0 & 0 & 0 & 0 & 0 & 0 & 0 & 0 & 0 & 0 & 0 & 0 & 0 & 0 & 1 & 0 & 0 & 0 \\
0 & 0 & 0 & 0 & 0 & 0 & -1 & 0 & 0 & 0 & 0 & 1 & 0 & 0 & 0 & 0 & 0 & 0 & 0 & 1 & 0 & 0 \\
0 & 0 & 2 & 0 & 0 & 0 & 0 & -3 & 0 & 0 & 0 & -2 & 0 & 0 & 0 & 0 & 0 & 0 & 0 & 0 & 1 & 0 \\
0 & 0 & 0 & -1 & 0 & 0 & 0 & 0 & 3 & 0 & 0 & 0 & 0 & 0 & -3 & 0 & 0 & 0 & 0 & 0 & 0 & 1
\end{array} \|
$$

and $\delta$ is identical with that in BI.

Four-Point Formula.

CI. $\left(p_{0}, q_{0}\right)=(0,0),\left(p_{1}, q_{1}\right)=(1,0),\left(p_{2}, q_{2}\right)=(0,1),\left(p_{3}, q_{3}\right)=(1,1)$.

$\alpha$ is the $1 \times 24$ matrix $\| F_{0}, F_{p_{0}}, F_{q_{0}}, F_{p p_{0}}, F_{p q_{0}}, F_{q q_{0}}, F_{1}, F_{p_{1}}, F_{q_{1}}, F_{p p_{1}}, F_{p q_{1}}, F_{a a_{1}}$, $F_{2}, F_{p_{3}}, F_{a s}, F_{p p_{3}}, F_{p q_{3}}, F_{q q_{3}}, F_{3}, F_{p_{3}}, F_{q_{3}}, F_{p p_{3}}, F_{p q_{3}}, F_{q q_{3}} \|$, 
$A$ is the $24 \times 24$ matrix

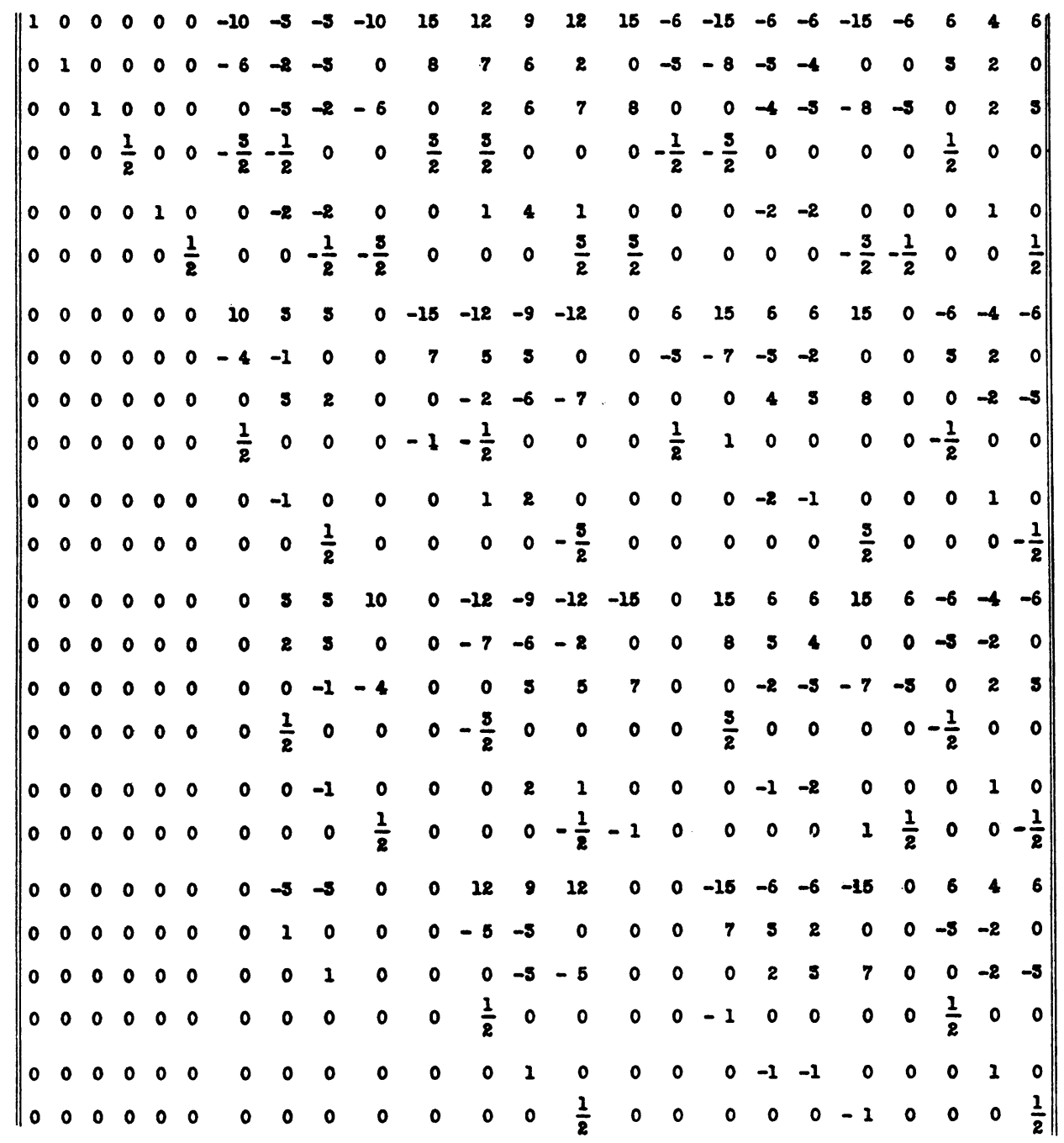

$\beta$ is the $1 \times 24$ matrix $\| 1, p, q, p^{2}, p q, q^{2}, p^{3}, p^{2} q, p q^{2}, q^{3}, p^{4}, p^{3} q, p^{2} q^{2}, p q^{3}, q^{4}, p^{5}$, $p^{4} q, p^{3} q^{2}, p^{2} q^{3}, p q^{4}, q^{5}, p^{5} q, p^{3} q^{3}, p q^{5} \|$,

$\gamma$ is the $1 \times 12$ matrix $\| F_{p p p p p p_{0}} / 720, F_{\text {ppppqa }} / 48, F_{p p q q q q_{0}} / 48, F_{\text {qqqqqa }} / 720$, $F_{\text {pppppppo }} / 5040, F_{\text {ppppppa }} / 720, F_{p p p p p q a} / 240, F_{\text {ppppqeq }} / 144, F_{\text {pppqeqa }} / 144, F_{\text {ppqeqeq }} / 240$, $F_{\text {peeseea }} / 720, F_{\text {qeqeque }} / 5040 \|$, 
$B$ is the $12 \times 30$ matrix

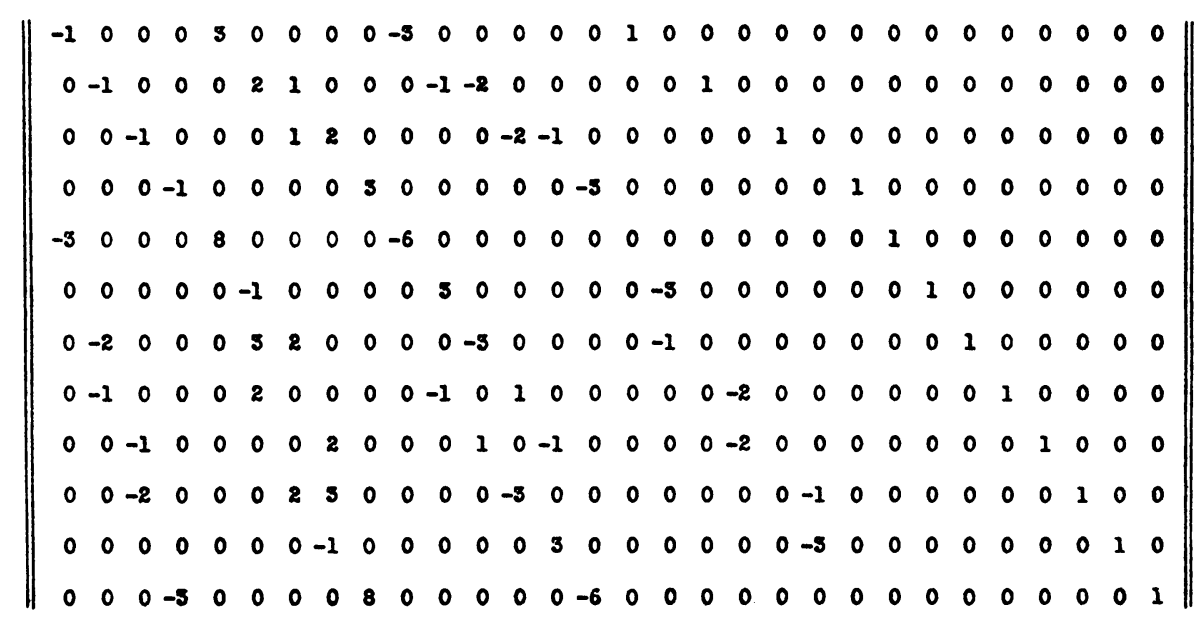

and $\delta$ is the $1 \times 30$ matrix $\| p^{3}, p^{2} q, p q^{2}, q^{3}, p^{4}, p^{3} q, p^{2} q^{2}, p q^{3}, q^{4}, p^{5}, p^{4} q, p^{3} q^{2}, p^{2} q^{3}, p q^{4}$, $q^{5}, p^{6}, p^{5} q, p^{4} q^{2}, p^{3} q^{3}, p^{2} q^{4}, p q^{5}, q^{6}, p^{7}, p^{6} q, p^{5} q^{2}, p^{4} q^{3}, p^{3} q^{4}, p^{2} q^{5}, p q^{6}, q^{7} \|$.

941 Washington Avenue

Brooklyn, New York 11225

1. H. E. SALZER \& G. M. KIMBRo, Tables for Bivariate Osculatory Interpolation over a Cartesian Grid, Convair Astronautics, $1958,40 \mathrm{pp}$. Note especially the introductory text, by the present writer, on pp. 1-23.

2. H. E. SALZER, Some New Divided Difference Algorithms for Two Variables, Proc. Sympos. Numerical Approximation (Madison, Wis., 1958), Univ. of Wisconsin Press, Madison, Wis., 1959, pp. 61-98. MR 21 \#960.

3. H. E. SALZER, "Divided differences for functions of two variables for irregularly spaced arguments," Numer. Math., v. 6, 1964, pp. 68-77. MR 29 \#2939. 